\title{
Advanced Review
}

\section{The CL\&Pol polarizable force field for the simulation of ionic liquids and eutectic}

\section{solvents}

\author{
Kateryna Goloviznina, Zheng Gong, and Agilio A. H. Padua* \\ Laboratoire de Chimie, École Normale Supérieure de Lyon 83 CNRS, 69364 Lyon, France \\ E-mail: agilio.padua@ens-lyon.fr
}

\begin{abstract}
Molecular dynamics simulation, and theoretical chemistry in general, have been central tools in the study of ionic liquids and eutectic solvents, both of which are fluid phases dominated by ionic interactions and strong hydrogen bonds. These systems are still relatively recent, so the amount of experimental information given their enormous diversity is still scarce. Computational studies have produced several important discoveries, besides helping in the interpretation of experimental findings. Here we review the latest developments in the models describing the interactions and conformations of ionic liquid phases with atomic detail, in particular polarizable force fields. In these all-atom models, the response of electron clouds to their electrostatic environment is represented by induced dipoles, therefore moving beyond the pair-wise additivity of fixed-charge force fields. Most of the conceptual framework to develop polarizable force fields has been available and implemented in major molecular dynamics codes, so the main challenge to develop a useful polarizable model for ionic liquids and eutectic solvents is how to handle their diversity in chemical structures and interactions, in order
\end{abstract}


to build a model that can represent solutions, mixtures and interfaces with materials, and that is not too difficult to extend to new systems. As usually, force field development is not only science: it's an art! The ingredients of successful force fields are often the result of pragmatism and heuristics, rather than mathematical complexity and absolutely rigorous physics (although retaining a sound physical basis in the parameters definitely helps). We focus on the CL\&Pol polarizable force field for ionic liquids, protic ionic liquids and eutectic solvents, improving on previous-generation fixed charge models. The CL\&Pol force field is built as a detailed but extendable and transferable model offering more reliable predictions of thermodynamic, structural and transport properties, which should contribute to the advancement of the field and towards the design of better solvents, electrolytes, lubricants, etc.

\section{Introduction}

Room-temperature ionic liquids have been a particularly active field of research for the past 20 years, ${ }^{1}$ in which molecular dynamics simulation was instrumental to predict and understand many physical and chemical properties. The atomistic force fields, or interaction potential models, are a fundamental ingredient of a molecular simulation study: if the underlying molecular model is not good, the results of the simulation will not be meaningful. Presently, at the forefront of this topic, several groups are actively developing force fields that incorporate polarization effects explicitly, thus representing the response of electron clouds to local electrostatic environments. Even highly detailed force fields are still orders of magnitude faster in computational terms than quantum mechanics, allowing us to access longer size and time scales. In ionic media, electrostatic forces need to be described carefully and the new polarizable models are more advanced than the traditional, fixed-charge models still in widespread use to study aqueous phases, organic compounds, biomolecules, interfaces, and of course also ionic liquids.

Ionic liquids (ILs) are salts formed by large organic ions, with asymmetric, flexible molec- 
ular structures and delocalized electrostatic charge, all of these features contribute to hinder crystallization and thus lower the melting point to near room temperature. The archetypal ionic liquid is butylmethylimidazolium bis(trifluoromethanesulfonyl)amide, $\left[\mathrm{C}_{4} \mathrm{C}_{1} \mathrm{im}\right]\left[\mathrm{Ntf}_{2}\right]$ (the anion is also known as TFSI): the cation has its charge delocalized over an aromatic 5-member imidazolium ring and has one nonpolar alkyl side chain, making it asymmetric; the anion has the charge delocalized over the $\mathrm{N}$ and the four $\mathrm{O}$ atoms, and is highly flexible with many accessible conformations. The extent of the liquid range, and the thermal and chemical stability of this IL are remarkable. Ionic liquids in which the ions are an acid-base pair (with the equilibrium largely displaced towards the ionic species) form the class of protic ionic liquids (PILs), of which an important example is ethylammonium nitrate (EAN). Hydrogen bonding is a key aspect in PILs (and can be also in "aprotic" ILs). The availability of these ionic fluid phases near room temperature opens up vast fields of application, as solvents for reactions, separations or for the synthesis of materials, as electrolytes in energy-storage devices, as lubricants, etc.

When speaking of ionic liquids we also include deep eutectic solvents (DES), ${ }^{2,3}$ a more recent class of environmentally acceptable solvents, which are mixtures of an organic salt with a molecular compound, typically with the role of H-bond donor. Several interesting organic salts that are abundant, low-cost and environmentally friendly, but solid, can be used to make liquids at room temperature taking advantage of the phase diagram of the mixture with a molecular compound, by staying close to the eutectic composition. The archetypal DES is a mixture of cholinium chloride with urea, both common and non-toxic compounds that are solid at room temperature, which yield a liquid mixture near the eutectic composition of roughly 1:2 molar ratio. The properties of the predominantly-ionic DES, like those of ILs, are determined to a large extent by Coulomb interactions and by H-bonds involving ions, with also contributions from non-polar groups. DES have many similar applications to those of ILs. From a molecular simulation standpoint, ILs $^{4-14}$ and DES ${ }^{15-19}$ can be modelled using the same framework and scales of description. 
Ionic liquids and DES are complex, structured phases, dominated by strong Coulomb interactions and with persistent medium-range ordering. As such, they pose several challenges to simulation methods and to the force fields, which have been highly successful tools to simulate aqueous solutions, organic liquids and biomolecules, and should in principle be equally applicable to ionic phases.

The slow dynamics (due to a relatively high viscosity) and long-range structural features of ionic liquids require simulations with consequent size and time scales (typically 6-10 nm and $10-50 \mathrm{~ns}$ ), significantly larger than those needed for typical molecular liquids, including aqueous or organic phases. In order to attain longer scales at lesser computational cost, coarse-grained (CG) models become invaluable, allowing the study of systems with persistent long-range ordering or with slow dynamics, for example the mesophases of thermotropic ILs (ionic liquid crystals). ${ }^{20}$ Several coarse-grained force fields have been developed for ionic liquids, in which typically one, or a few, CG sites ("beads") bearing an electrostatic charge represent the ionic head-groups, and each CG site on the side chains represents a few $-\mathrm{CH}_{2}-$ units. Most $\mathrm{CG}$ force fields are specific to certain families of $\mathrm{ILs}^{21,22}$ and are not easy to combine with other models, but the Martini force field ${ }^{23}$ which is fragment-based, has been recently extended to ILs, ${ }^{24}$ providing a tool to simulate many different systems, since the Martini force field is designed to allow mixing of fragments.

Coarse-grained models retain the essential physics of a molten salt, namely the charge ordering an the ionic correlations. However, these models average-out many molecular degrees of freedom, hiding certain details of specific interactions, such as H-bonding sites, electrostatic charge distributions or conformational features. So-called united-atom mod$\mathrm{els}^{25}$ only hide a few degrees of freedom corresponding to $\mathrm{H}$ atoms, which are fused into the heavy atoms, thus keeping many important features. In our view, keeping a high level of detail is important for many studies, namely to design ionic liquids as solvents, lubricants or electrolytes, through variations in their molecular structures that lead to subtle effects and finely-tuned properties. This is one of the aspects that make room-temperature ILs 
interesting in terms of their physical chemistry, and also for many applications.

On the smaller side of the multi-scale approach, in which the electronic structure is computed using quantum mechanics, only very small systems can be studied (a few dozen ion pairs) and during short trajectories. These ab initio molecular dynamics methods are very powerful to study local, quasi-chemical effects but do not reach the scales needed to evaluate energetic, structural and dynamic properties of the ionic liquid phases or their interfaces. Therefore, at present, all-atom molecular dynamics strikes the best compromise between computational cost, level of detail, and size and time scales attainable. ${ }^{26}$

Since only very little experimental information was available on ionic liquids and DES, as this field evolved simulation has contributed to discover interesting features about these systems, as well as serving as a tool to interpret macroscopic properties from the molecular level. ${ }^{26}$ Also, it makes sense to benefit from advanced computational methods to select fewer, and better targeted, experiments. For example, the nanoscale segregation between polar and non-polar domains in ILs, where medium-length alkyl side chains are segregated from charged head-groups due the strong cohesion between the latter, was seen in all-atom simulations ${ }^{27}$ and later confirmed by X-ray scattering. ${ }^{28}$ This heterogeneous nature of ILs has important consequences for solvation. Our understanding the correlations in ionic motions, concerning how conductivity and diffusion are related, was also largely improved through MD simulation studies. ${ }^{29}$ Understanding ionic transport is key for the use of ILs as electrolytes. The design of task-specific ionic liquids for $\mathrm{CO}_{2}$ capture is another example of a research effort in which experiments and computational chemistry made progress together. ${ }^{30}$ The field of DES is newer, and simulating DES is in certain aspects more complex: they are by definition multicomponent mixtures containing both ions and neutral molecules, with strong H-bonds involving ions, which are challenging to model. When compared to ILs, the species in DES have more freedom to create solvation environments with local compositions that differ from the average composition. In purely ionic media this is more difficult because of the constraint of electroneutrality. In DES, simulations have shown local solvation environments 
that enhance the solubility of nonpolar, hydrophobic compounds. ${ }^{31}$

Because of the enormous variety of chemical structures (and compositions) of ILs and DES, and in the logic of using computational methods to identify and design novel solvents or electrolytes, molecular force fields should aim for a wide applicability, both in terms of the functional groups they can represent and the properties that can be computed. Traditional fixed-charge force fields for organic compounds, such as OPLS-AA, ${ }^{32}$ AMBER/GAFF ${ }^{33}$ or CHARMM/CGenFF, ${ }^{34}$ offer the advantage of vast parameter sets covering many functional groups, and also of a degree of transferability that allows them to be ported to new molecules or ions with just reasonable effort, following well-documented parameterization procedures. Unfortunately, this class of fixed-charge models proved unable, in general, to represent both structural and dynamic properties of ILs correctly, likely because they were not developed for ionic phases. When using integer ionic charges, dynamics is systematically predicted to be too slow when compared to experiment, ${ }^{35,36}$ roughly by one order of magnitude in diffusion coefficient or viscosity. The heat of vaporization seems also to be somewhat overestimated. Scaling-down the ionic charges by a factor of about $0.8^{37}$ can improve the sluggish dynamics by reducing the ionic cohesive energy. Physical arguments in favor of scaling charges include accounting for charge transfer and for effective polarization effects, ${ }^{38}$ the the basis of these arguments in bulk liquid phases are not totally sound. Unfortunately, with scaled charges densities tend to become too low, by up to $5 \%$, and in mixtures and solutions the balance between solute-solute, solute-solvent and solvent-solvent interactions may be modified, compromising the prediction of solubility, miscibility and phase behaviour. ${ }^{39}$ Studying mixtures and solutions involves calculations for molecular or ionic species in different environments and a charge scaling factor optimized for a certain state may not be transferable to different environments. ${ }^{38}$ Charge scaling also affects dielectric properties. ${ }^{40}$

These shortcomings have motivated the development of more sophisticated force fields, which include to some extent a representation of electronic degrees of freedom through explicit polarization effects, thus moving beyond pairwise-additive interaction models. This 
representation of electron clouds is, of course, hugely simplified when compared to electronic structure calculations (such as density functional theory) but the computational cost is kept of the order of traditional molecular dynamics (MD) with additive, fixed-charge models. These developments for ILs have followed the evolution of polarizable force fields for organic compounds and biomolecules, namely the CHARMM family of models, ${ }^{41}$ and they have been the subject of a recent review. ${ }^{42}$ Here we will give an account of additional, newer developments in polarizable force fields that extend their applicability to protic ionic liquids and to DES, both the site of strong H-bonds involving densely-charged ions. It is curious that along the way, "old" tricks from simulation of high-temperature molten salts ${ }^{43}$ have been brought in to solve some technical issues with the polarizable models. The developments reviewed here aim for a general, transferable model, suitable to handle the huge variety of ILs and DES, so that it can be used as a tool in molecular design of novel materials.

\section{Representing Polarization Explicitly}

Polarization (or induction) terms can be incorporated in MD force fields through several methods. Adding point induced dipoles ${ }^{44-46}$ or Drude induced dipoles ${ }^{41,47-50}$ to atomic sites are nearly equivalent methods, based on different ways to materialize the induced dipoles. Point induced dipoles are just vector quantities assigned to the atomic sites, whereas Drude induced dipoles are formed by two particles of opposite charge connected by a spring. The response of the induced dipoles to the electrostatic environment is determined by the polarizability of the atomic sites, and so polarizable force fields have one additional type of parameter, but fortunately with a clear physical meaning. Only minor deviations between point and Drude induced dipoles have been documented, and that for highly polarizable

atoms. ${ }^{51}$ Using fluctuating point charges on atomic sites ${ }^{52-56}$ provides an alternative method to represent polarization, with some drawbacks, such as inability to describe out-of-plane polarization and not being easy to mix polarizable and non-polarizable particles, and some 
advantages, namely that no additional particles are required. With fluctuating charges, electronegativity and hardness parameters are used to compute on-the-fly the atomic partial charges, which are not set a priori, and so charge-equilibration models can naturally render charge transfer between molecules. However, taming charge transfer requires additional mechanisms. ${ }^{57,58}$ Fluctuating-charge models have not been developed to cover as many molecular structures as induced-dipole models. The implementation of the Drude induced dipole method in widely used codes, including CHARMM, ${ }^{49}$ NAMD,${ }^{59}$ Gromacs, ${ }^{60}$ LAMMPS ${ }^{61}$ and OpenMM, ${ }^{62}$ makes this technique the most obvious choice for ionic liquids and DES.

Polarizable force fields have been proposed for some ionic liquids, ${ }^{63-66}$ and almost none for DES, but only a few efforts to develop systematic and general polarizable models have been undertaken. ${ }^{67-71}$ The APPLE\&P force field by Borodin ${ }^{67}$ was pioneering, with excellent predictions of thermodynamic, structural and transport properties of ILs. But extending and combining this force field to model other compounds or materials is difficult because of the uncommon functional form (exponential-6 potential and combining rules). Besides, this is a proprietary model whose parameters are not available in the open literature. Among recent Drude-based models, the SAPT-FF force field ${ }^{70,72}$ and the transferable CL\&Pol force field ${ }^{71,73,74}$ incorporate information from accurate quantum calculations using symmetry-adapted perturbation theory (SAPT), a suitable quantum chemistry method to obtain interaction energies and their decomposition into repulsive, electrostatic, induction and dispersion terms. The SAPT-FF model ${ }^{70,72}$ for ILs is developed from scratch with all intermolecular parameters fitted to SAPT energies. This model has been extended with a study of polyethylene oxide in an $\mathrm{IL}^{75}$ and recently of urea, ${ }^{76}$ which point towards an eventual extension to DES. The functional form of this force field consists of van der Waals interactions modelled by an exponential Born-Mayer repulsive term and a series of $C_{n} / r^{-n}$, $n=6,8,10,12$ powers $^{77}$ for the dispersion term. The case-by-case and costly SAPT parameterization, as well as the incompatibility of the functional form to that of widely used force 
fields are obstacles to transferability and a broad usage of this force field.

The CL\&Pol model ${ }^{71,73,74}$ presented in this review is built according to a different strategy, favouring generality and transferability. It was developed from the existing fixed-charge CL\&P model, ${ }^{7,13}$ one of the most widely used for ILs, that had been parameterized following the philosophy and functional form of OPLS-AA (and it is also compatible with AMBER/GAFF). As such, the CL\&Pol model is relatively easy to extend, with applicability already going beyond ILs and DES, having been used to study for example alkalimetal electrolytes, dissolved gases, dyes and interfaces with nanomaterials. The qualities of the CL\&Pol force field were demonstrated in the calculation of thermodynamic, structural and transport properties, yielding significant improvements when compared to the previous generation of fixed-charge models. The CL\&Pol force field, including parameter database, examples and system-building tools for major codes, is available at public code repositories (https://github.com/paduagroup).

\section{Building the CL\&Pol Force Field}

The CL\&Pol polarizable force field is built on the basis of the CL\&P fixed-charge model following several steps, illustrated in Fig. 1:

1. Adding Drude induced dipoles derived from atomic polarizabilities.

2. Scaling down the well-depth of the van der Waals interactions to avoid double-counting of induction effects (which were implicit in Lennard-Jones terms of the CL\&P model). This modification is performed based on SAPT calculations, but a computationally light scheme was developed to enable easy extension.

3. Slightly adjusting atomic diameters to improve the calculation of density.

The intramolecular bonded parameters and the atomic partial charges are taken from the CL\&P values. The intramolecular terms describing covalent bond stretching and va- 


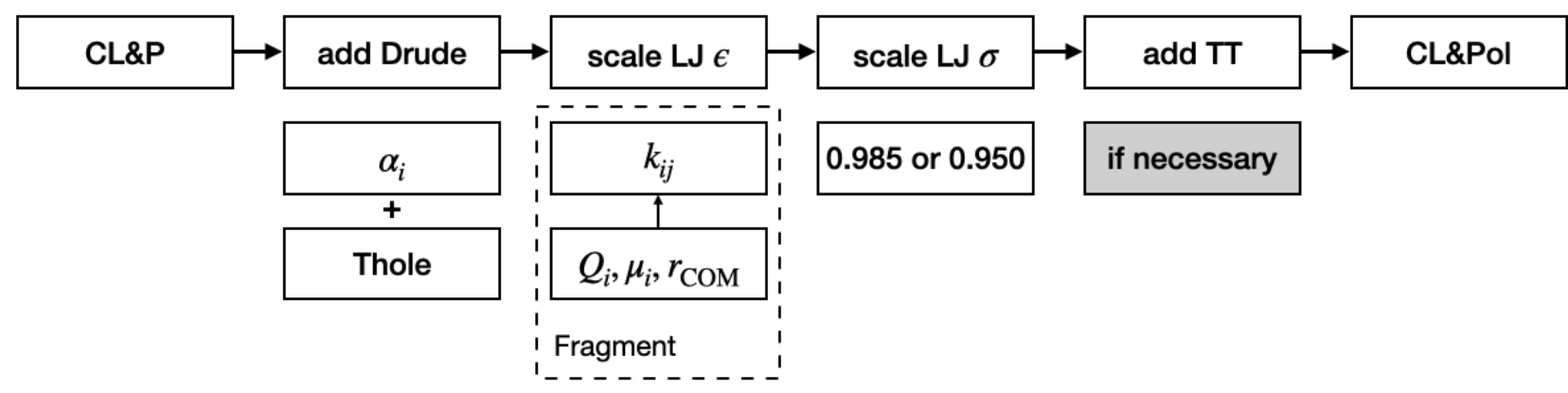

Figure 1: Layout of the procedure to obtain the CL\&Pol force field from the original CL\&P fixed-charge model.

lence angle bending are taken whenever available from the extensive OPLS-AA ${ }^{32,78}$ and AMBER $/ \mathrm{GAFF}^{79}$ parameter sets. If not available, the force constants and equilibrium geometries are calculated using the quantum chemical methods recommended for these force fields, thus maintaining compatibility with many molecular compounds. Thermodynamic and transport properties computed from condensed-phase MD, by averaging over long trajectories of many nanoseconds, are not sensitive to details of vibrational modes (whose periods are 6 orders of magnitude shorter). Certain ideal-gas properties would be sensitive to details of bonded terms. The torsion (or dihedral angle) potentials, which affect conformations, and the non-bonded Lennard-Jones (LJ) and Coulomb terms are, on the other hand, critical for condensed-phase simulations. So-called "proper" torsion potentials act between groups of 4 atoms, 1-2-3-4 bonded through 3 bonds, rotating around the central 2-3 bond, and are key to describe conformations. "Improper" torsion potentials are applied to groups of 4 atoms of which 3 of them are bonded to a central, planar atom, usually the 3rd, in order to stiffen out-of-plane bending of $\mathrm{sp}^{2}$ carbon or nitrogen atoms, for example.

The dihedral angle potentials do not determine the conformations alone, since atoms belonging to the same molecule also interact through LJ and Coulomb potentials if they are sufficiently far apart (4 or more bonds), which represents steric hindrance effects and prevents overlaps. Therefore, an interdependence exists between the torsion potentials and the LJ and Coulomb parameters. The OPLS-AA procedure requires, first, that charges and 
LJ parameters be obtained, and only then dihedral angle coefficients be fitted to quantum mechanically calculated torsion energy profiles. The torsion parameters of both CL\&P and CL\&Pol are obtained in this consistent manner ${ }^{8,78}$ in an attempt to describe ion conformations accurately.

\subsection{Drude Induced Dipoles}

A Drude induced dipole is formed by two point charges of opposite sign, a positively-charged Drude core (DC), at the center the atomic site, and a negatively-charged Drude particle (DP), connected to its core by a harmonic spring with an equilibrium distance of zero. The pair of charges gives rise to an induced dipole under an external electrostatic field, with magnitude determined by the Drude charges and by the force constant of the spring,

$$
\alpha=\frac{q_{D}^{2}}{k_{D}}
$$

We opt, as in the CHARMM polarizable force field, ${ }^{49}$ to set the force constant of the DCDP harmonic bond to $k_{D}=4184 \mathrm{~kJ} \mathrm{~mol}^{-1}$ and obtain the Drude charge from the atomic polarizability. Atomic polarizabilities for ionic liquids derived from first principle calculations are available in the literature. ${ }^{80,81}$ Because of the small atomic mass comparable to the mass of the DP and the low polarizability (low electron density), hydrogen atoms are treated as non-polarizable, with their polarizability merged onto the heavy atoms to which they are bound.

In a MD trajectory, the degrees of freedom (DOF) associated with Drude dipoles can be relaxed iteratively to their equilibrium positions at each time step, but this self-consistent relaxed-Drude method is slow. A faster alternative is to integrate the equations of motion of the DP by assigning a mass to them (usually $0.4 \mathrm{u}$, which is subtracted from the respective DC). The aim is for this extended Lagrangian method to generate a trajectory as close as possible to that of the relaxed Drudes, so in order to prevent kinetic energy draining into 
the DC-DP DOF, the relative motion of the DP with respect to their DC is thermostated at very low temperature, ${ }^{49}$ typically of $1 \mathrm{~K}$, with the remaining DOF being thermostated at the desired temperature. Therefore, the implementation of the Drude model in MD codes requires a dual-thermostat scheme, which has been implemented in several MD packages. ${ }^{59-62}$ It was recently shown that heat flow to the cold Drude DOFs may lead to unbalanced temperatures between intramolecular and translational center-of-mass DOF. ${ }^{82}$ To correct this bias, a triple temperature-grouped Nosé-Hoover thermostat has been implemented in the OpenMM ${ }^{82}$ and LAMMPS ${ }^{74}$ codes. This improved thermostating scheme provides more accurate dynamic and structural quantities.

\subsection{Preventing the Polarization Catastrophe}

In a Drude polarizable force field such as CL\&Pol the electrostatic potential between the induced dipoles includes a short-range damping function:

$$
U_{e l s t}\left(r_{i j}\right)=\sum_{i} \sum_{j \neq i} \frac{q_{i} q_{j}}{r_{i j}}+\sum_{i} \sum_{j} T_{i j}\left(r_{i j}\right) \frac{q_{i} q_{D, j}}{r_{i j}}+\sum_{i} \sum_{j \neq i} S_{i j}\left(r_{i j}\right) \frac{q_{D, i} q_{D, j}}{r_{i j}}
$$

where $T_{i j}$ and $S_{i j}$ are short-range damping functions, plotted in Fig. 2. These damping functions represent smearing of the point charges at short range, which has a physical sense ${ }^{61,83-86}$ when describing the interactions of electron clouds, and also help to prevent instabilities in the trajectories due to excessive correlations between nearby dipoles, or to rare events in which DP are captured by nearby positive particles, for example DC of other atoms, causing a "polarization catastrophe".

We use Thole damping functions, $S_{i j}{ }^{87}$ (3), controlled by the atomic polarizabilities $\alpha$ 


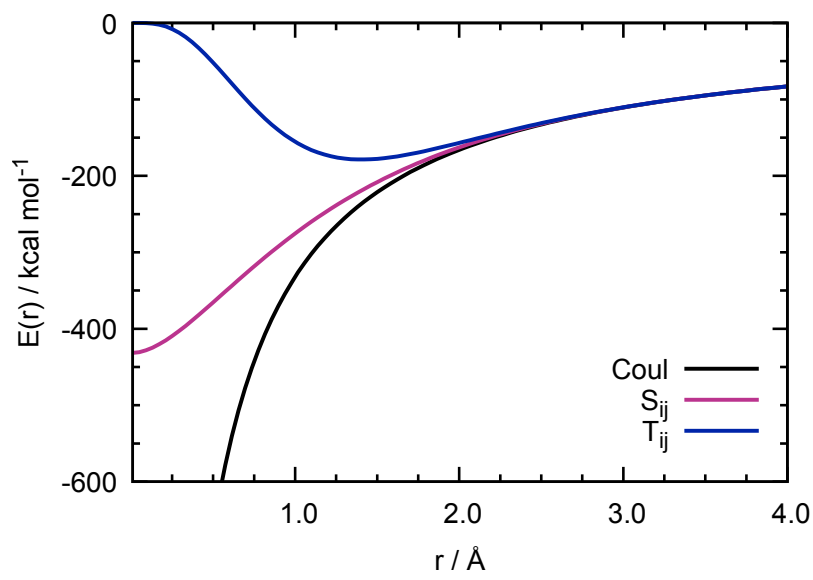

Figure 2: Short-range damping of Drude dipole-dipole interactions using a Thole function, $S_{i j}\left(a=2.6, \alpha=1.5 \AA^{3}\right)$ and of charge-Drude dipole interactions using Tang-Toennies functions, $T_{i j}\left(b=4.5 \AA^{-1}, c=1.0\right)$.

and a universal parameter $a_{i j}=2.6 .^{84}$

$$
\begin{aligned}
S_{i j}\left(r_{i j}\right) & =1-\left(1+\frac{s_{i j} r_{i j}}{2}\right) \exp \left(-s_{i j} r_{i j}\right) \\
s_{i j} & =\frac{a_{i j}}{\left(\alpha_{i j}\right)^{1 / 3}}=\frac{\left(a_{i}+a_{j}\right) / 2}{\left[\left(\alpha_{i} \alpha_{j}\right)^{1 / 2}\right]^{1 / 3}}
\end{aligned}
$$

For most aprotic ionic liquids, Thole damping is sufficient to avoid instabilities. But in strongly H-bonded systems, including protic ionic liquids and DES, and particularly in the presence of densely-charged ions such as alkali metal cations or halogens, the interactions between certain charges and induced dipole need also to be damped (or smeared) at short range. ${ }^{74}$ In the CL\&Pol force field, this damping of charge-dipole interactions is used only to prevent instabilities of simulations. Inspired by the MD studies of molten salts ${ }^{43,66,88-90}$ we used an adaptation of the Tang-Toennies ${ }^{91}$ (TT) dispersion damping function,

$$
T_{i j}\left(r_{i j}\right)=1-c_{i j} \cdot \exp \left(-b_{i j} r_{i j}\right) \sum_{k=0}^{4} \frac{\left(b_{i j} r_{i j}\right)^{k}}{k !}
$$

where the parameters $b_{i j}$ and $c_{i j}$ are set empirically to 4.5 and $1.0 .{ }^{74}$ The TT damping function serves as a safeguard against events causing trajectory instabilities, preventing DP 
from being captured by nearby positive atoms (Fig. 3) without affecting properties of systems in normal circumstances.

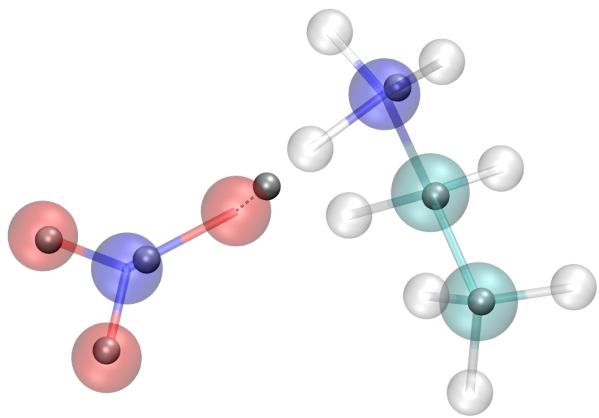

Figure 3: Illustration of the polarization catastrophe in ethylammonium nitrate, a protic IL. The DP on the $\mathrm{O}$ atom of the anion leaves its $\mathrm{DC}$ and is pulled towards the $\mathrm{H}$ atom of the cation head group. Hydrogen atoms are not polarizable and so do not carry a DP.

\subsection{Transferability by a fragment approach}

The CL\&Pol force field is designed to be extendable to new compounds with as much transferability of parameters as possible. Large ions are decomposed into smaller fragments for the purpose of deriving the force field parameters, and the chemical functional groups of the fragments are kept as much as possible unchanged across molecular or ionic entities. Typically, an ion is represented by a charged head group and one or several neutral side chains, for example, 1-butyl-3-methylimidazolium is split into 1-ethyl-3-methylimidazolium, over which the positive charge is distributed, and butane serves as template to the atoms in the side chain that are sufficiently far from the charged head-group. ${ }^{73}$ We keep the ethyl group in the first fragment to emphasise that the imidazolium ring affects partial charges on atoms up to two bonds along the chain. ${ }^{7}$ On the contrary, if the charge is not spread over a conjugated $\pi$-system, but localized on a particular atom with several side chains attached to it, such as in quaternary ammonium or phosphonium cations, a fragment with short methyl groups such as tetramethylammonium is used. Often anions, which are typically much smaller than cations in the most usual ILs, are kept as entire ions. Naturally, in cases such as alkylsul- 
fonatea or tosylate, long-chain carboxylates or phosphates, separate fragments are defined for the negative head group and for the neutral side chains or groups.

Once the general procedure is established, a balance between the number of fragments and their size should be kept, in order to avoid over-parameterization of the force field. Some cases merit a special treatment. Thus, in ethylammonium nitrate, an important protic ionic liquid, and in choline chloride, present in many DES, the cations are treated entirely and

parameterized specifically, since they are still relatively small and this makes the force field more accurate for these compounds. In more general IL and DES families it is much more productive to use a transferable fragment approach, even if some specific details are less well reproduced.

\subsection{Separating Polarization from Dispersion Interactions}

The parameters describing van der Waals interactions in CL\&Pol are taken from the nonpolarizable CL\&P force field for ionic liquids, or from OPLS-AA for molecular compounds. In these fixed-charge models, polarization effects are already present, albeit described implicitly, lumped with dispersion terms in the non-bonded Lennard-Jones potential. Now in the CL\&Pol force field, polarization (induction) effects are accounted for explicitly through the Drude induced dipoles. Therefore, the original CL\&P LJ parameters need to be rescaled to remove double counting of polarization terms, so that in CL\&Pol the LJ terms represents only London dispersion. We perform this through a scaling factor, $k_{i j}$, which is applied to the well-depth, $\epsilon$, of the LJ potential, corresponding to the fraction of dispersion contribution in the sum of dispersion and induction energies,

$$
k_{i j}=\frac{E_{\text {disp }}}{E_{\text {disp }}+E_{\text {ind }}}
$$

This scaling factor is evaluated per fragment, and functional groups present in the same ion can have different values, depending on the fragment they belong to. We aimed to have the 
best possible resolution between the different terms making up the intermolecular energies, specially the induction and dispersion components, so we chose SAPT, ${ }^{92,93}$ an expensive method which doesn't suffer from basis-set superposition errors, to evaluate dimer interaction energies. ${ }^{71,73}$ Only dimers of fragments that can be found in first coordination shells, namely cation-anion, cation-neutral, anion-neutral and neutral-neutral, are considered. The nonbonded interactions between ions of the same charge are not modified due to the larger separations (second solvation shell) arising from electrostatic repulsion. The values of $k_{i j}$ are nearly 1.0 for apolar-apolar fragment dimers (e.g. two butane molecules representing alkyl chains) because the induction component is small compared to the dispersive one. When polar or charged fragments are involved, the values of $k_{i j}$ can be 0.4 or even less, meaning that the polarization terms are dominant compared to dispersion.

The high computational cost of SAPT calculations prompted us to devise a general scheme to predict the scaling factors on the basis of accessible molecular quantities, such as total charges, polarizabilities and dipole moments of the fragments. ${ }^{71}$ Based on the classical equations of London dispersion and Debye induction, the scaling factor can be expressed as

$$
k_{i j}=\left(1+c_{0} r_{i j}^{2} \frac{Q_{i}^{2} \alpha_{j}+Q_{j}^{2} \alpha_{i}}{\alpha_{i} \alpha_{j}}+c_{1} \frac{\mu_{i}^{2} \alpha_{j}+\mu_{j}^{2} \alpha_{i}}{\alpha_{i} \alpha_{j}}\right)^{-1}
$$

where the $\alpha$ are the polarizabilities of the fragments, $Q$ their net charges, $\mu$ their dipole moments. The distance $r_{i j}$ is the equilibrium separation of a given fragment pair, and $c_{0}=0.25$ and $c_{1}=0.11$ are adjusted coefficients to a set of SAPT-derived $k_{i j}$ values covering charged and neutral fragments, ${ }^{71}$ as shown in Fig. 4. The root-mean square error does not exceed 0.08 , which we find quite good, since conformations and local geometry minima also cause uncertainties in the SAPT values.

Recently, the scheme was applied to dimers composed of new tetramethylphosphonium $\left(\mathrm{P}_{1111}^{+}\right)$and cholinium $\left(\mathrm{Ch}^{+}\right)$cations, tetracyanoborate $\left(\mathrm{TCB}^{-}\right)$and 2,2,2-trifluoro-N-(trifluoromethylsulfony (TSAC ${ }^{-}$) anions, dimethyl ether $\left(\mathrm{Me}_{2} \mathrm{O}\right)$ and tetramethylsilane (TMS) molecules, and the ro- 


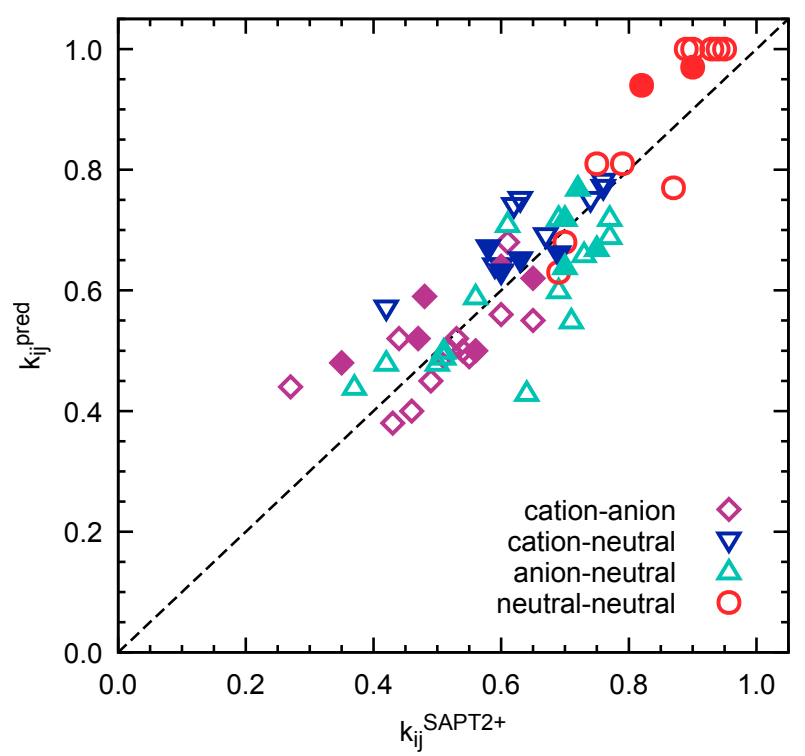

Figure 4: Predicted and SAPT-calculated $k_{i j}$ scaling factors. Empty points are those used in the original CL\&Pol development, ${ }^{71}$ whereas filled points are more recent involving new fragments.

bustness of the prediction is demonstrated by the reliability of our approach when extending it to new structures.

Mixing CL\&Pol with the other polarizable force fields, for example the SWM4-NDP water model ${ }^{94}$ or a polarizable $\mathrm{CO}_{2}$ model, ${ }^{95}$ is rather straightforward. But in these cases, only the influence of the already polarizable molecules (water or $\mathrm{CO}_{2}$ ) on the CL\&Pol fragments should be taken into account to scale the LJ terms (coming from CL\&P),

$$
k_{i j}=\left(1+c_{0} r_{i j}^{2} \frac{Q_{i}^{2}}{\alpha_{i}}+c_{1} \frac{\mu_{i}^{2}}{\alpha_{i}}\right)^{-1}
$$

where the $i$ index corresponds to the already polarizable molecule. In the case of a water molecule, for example, this expression depends only on its dipole moment and molecular polarizability, ${ }^{94}$ resulting in a value of 0.72 for any dimer formed with SWM4-NDP water.

The procedure described so far to generate the CL\&Pol parameters, by adding Drude induced dipoles and scaling down the well-depths of the LJ potentials, was seen to lead to systematically slightly lower densities by a few percent on the training set of ionic liquids. ${ }^{71}$ 
This does not seem to be a spurious effect of the dual thermostat used to handle the Drude DOF, as has been reported in the literature ${ }^{82}$ since for the CL\&Pol force field both classical and temperature-grouped Nosé-Hoover thermostat give comparable density deviations ${ }^{74}$ as seen in Fig. 5. Therefore, we interpret the lower densities as due to the procedure to parameterize the CL\&Pol model, and opted to nudge the values of the LJ diameters $\sigma$ by a universal factor of 0.985 , i.e. a $1.5 \%$ correction, in order to compensate for the density deviations. ${ }^{71}$ For some particular cases, such as ammonium and phosphonium cations with several long alkyl side chains, for example trihexyltetradecylphosphonium $\left(\mathrm{P}_{6,6,6,14}+\right)$, the correction needs to be more consequent, with $\sigma$ scaled by 0.950 in order to keep density deviations within $-2.5-3.0 \%$. This seems to be an issue arising from the very long side chains rather than from the ionic or polar fragments, and likely could benefit from sourcing the alkyl chain parameters from a native polarizable force field for alkanes.

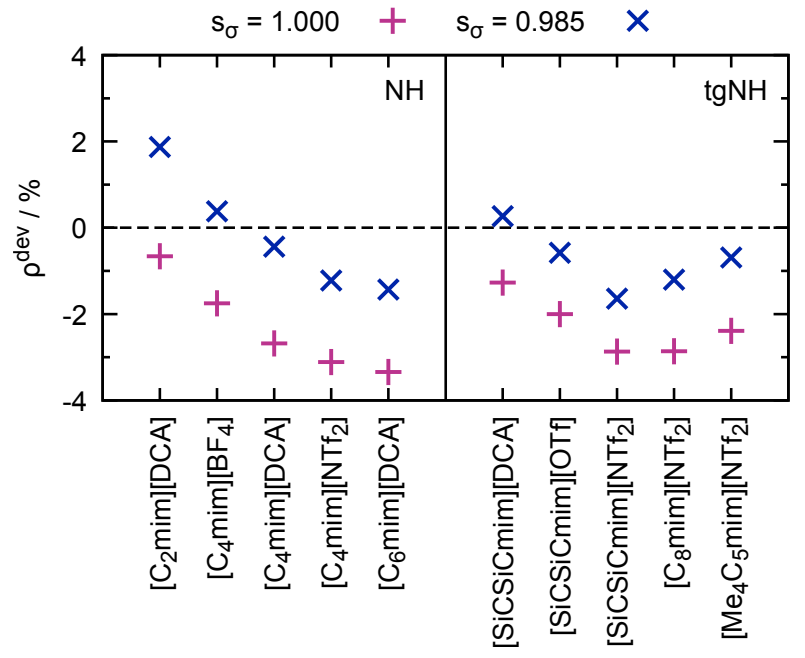

Figure 5: Predicting the density of ILs before and after LJ $\sigma$ correction by 0.985 . NH denotes the dual Nosé-Hoover thermostat, tgNH the temperature-grouped Nosé-Hoover thermostat; $\mathrm{SiCSiC}$ corresponds to dimethyl((trimethylsilyl)methyl)silyl)methyl, $\mathrm{Me}_{4} \mathrm{C}_{5}$ to 2,2,4,4-tetramethylpentyl side chains in imidazolium cations. 


\section{Modelling Difficult H-bonds in Protic ILs and DES}

When modelling cholinium chloride $(\mathrm{ChCl})$ based DES and other strongly H-bonded system with halide ions, we observed a notable shortening of $\mathrm{O}-\mathrm{H} \cdots \mathrm{Cl}$ distance (to $1.7 \AA$ ), a density overestimation (3.5\%) and "freezing" of the system, meaning the strong H-bonds involving densely-charged ions were not modelled correctly. The absence of a repulsive core on a hydrogen atom was allowing the positive charge of the $\mathrm{H}$ to approach $\mathrm{Cl}^{-}$and its $\mathrm{DP}$, even when using the TT damping function. Note that $\mathrm{H}$ atoms bonded to $\mathrm{O}$ or to $\mathrm{N}$ in the OPLS-AA force field do not carry their own LJ sites; they are simply point charges embedded in the LJ site of the corresponding heavy atom (this is also the case in many water models). During the development of CL\&Pol, attempts including a small LJ site on $\mathrm{H}$ atoms were made, to prevent H-bonds from becoming too short. But a satisfactory compromise between preventing instability in the Drude dipoles and maintaining correct structural features was not found. This led us to revise the source of the CL\&P chloride parameters, which had been obtained from the crystal structures for the few available ILs more than 15 years ago. ${ }^{7,9}$ At the time this was thought to better represent the fully ionic nature of ILs than parameters for ions derived from hydration properties. But for strongly H-bonded systems, chloride parameters from OPLS-AA, which were obtained from aqueous solutions, ${ }^{96}$ much improve the situation. Indeed in OPLS-AA $\mathrm{Cl}^{-}$has a larger LJ diameter $(4.41 \AA$ instead of $3.65 \AA$ in the initial CL\&P). Adopting OPLS-AA as a source of parameters for halides leads to a good prediction of equilibrium and transport properties of DES, such as cholinium chloride + urea, cholinium chloride + ethyene glycol and tetrabutylammonium bromide + ethylene glycol, providing us with a suitable polarizable force field for DES.

Quite similarly, in protic ionic liquids such as ethylammonium nitrate (EAN), a similar issue related to the shortening of $\mathrm{N}-\mathrm{H} \cdots \mathrm{O}$ distance was seen. There are three "nakedcharge" $\mathrm{H}$ atoms in the ammonium head group and their interactions with a nitrate anion led to an extremely short $\mathrm{N}-\mathrm{H}$. . O distance of $1.2 \AA$, comparable in length to an intramolec-

ular $\mathrm{N}-\mathrm{H}$ bond, whereas ab initio calculations give a $\mathrm{N}-\mathrm{H} \cdots \mathrm{O}$ distance of $1.9 \AA .{ }^{97}$ As said 
above, EAN is such an important liquid due to its network of hydrogen bonds, and this status grants a specific treatment in force field development (much like for water). Coincidentally, there have been contradicting analyses in the literature about the nature of the H-bonds in EAN. In one view, based on the empirical potential refinement method from X-ray and neutron structure factors, a number of authors ${ }^{98-100}$ postulated bent $\mathrm{N}-\mathrm{H} \cdots \mathrm{O}$ bonds, with an $\mathrm{O}$ atom from nitrate shared between several ammonium $\mathrm{H}$. A different view, of linear H-bonds involving one ammonium $\mathrm{H}$ was advanced by other groups, ${ }^{97,101,102}$ from DFT trajectories in condensed phase. The two patterns are shown in Fig. 6. The interpretation of the H-bond geometry from structural data seems to be highly sensitive to the underlying atomistic model, ${ }^{103}$ so this is an open question. Going back to the extension of CL\&Pol to EAN, correct H-bond distances are obtained by increasing $\sigma_{\mathrm{NO}}$ slightly. ${ }^{74}$ This leads to density, viscosity, diffusion coefficients, and also X-ray and neutron structure factors from polarizable MD simulations that are in good agreement with experimental data. ${ }^{74}$ It also produces linear H-bonds, supporting this hypothesis about the structure of EAN.
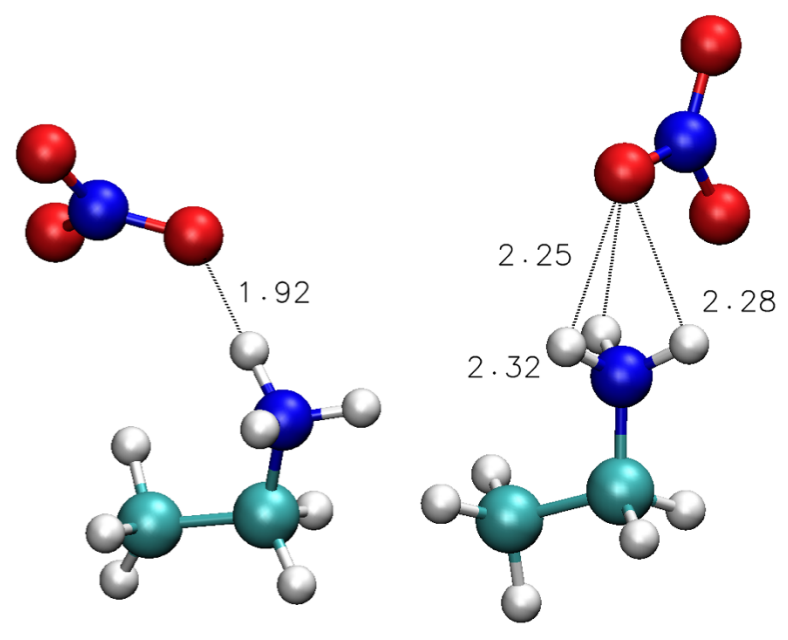

Figure 6: Two hydrogen bonding patterns in ethylammonium nitrate. A linear $\mathrm{N}-\mathrm{H} \cdots \mathrm{O}$ bond obtained from QM calculations in condensed phase ${ }^{97,101,102}$ and the CL\&Pol force field ${ }^{74}$ (left) and a bent, shared $\mathrm{N}-\mathrm{H} \cdots \mathrm{O}$ bond obtained with the empirical potential refinement method from X-ray and neutron scattering ${ }^{98-100}$ (right). 


\section{Ionic liquids at charged interfaces}

Many ionic liquids and DES are suitable as electrolytes, some with wide electrochemical windows, so among the first obvious applications one finds metal and semiconductor electrodeposition processes, ${ }^{104}$ and energy storage devices, ${ }^{105,106}$ both batteries and supercapacitors. Modelling these systems is more difficult than bulk liquid phases because of the interface with the electrode material, ${ }^{107}$ which means describing interactions between a solid and a liquid, often complicated because solid and liquid-state modelling may use incompatible functional forms in the force fields. On top of this, a correct description of electrostatic effects is essential to describe electrodes and their interfacial layers. The surface charges on the electrode can be modelled simply by assigning a fixed charge distribution, from which the electrostatic potential is calculated integrating Poisson's equation (this is the analogue of a fixed-charge molecular force field) or, in a more realistic manner, by imposing a potential and computing the corresponding atomic charge distribution, ${ }^{108,109}$ which fluctuates in response to the ions of the electrolyte and therefore polarization effects are explicit. Constant-potential boundary conditions at conductors can also be modelled using the image charge convention. ${ }^{110}$

Given the nature of the electrodes and of the IL electrolytes, it would be interesting to treat both using polarizable models, to representing more faithfully the interactions, ordering and dynamics in the interfacial layers, that are not yet fully understood. ${ }^{111}$ But in most published studies using simulation, the two partners, electrode material or ionic liquid, are not treated with equivalent levels of detail, thus some important effects may be missed. We think in the future, with the availability of polarizable force fields such as CL\&Pol, the community working on electrochemical devices will opt to model all-atom, fully polarizable systems. The CL\&Pol force field has been used to study substituted imidazolium dicyanamide ILs at charged interfaces of molybdenum disulfide, $\mathrm{MoS}_{2}$, a two-dimensional material of the family of transition metal dichalcogenides. $\mathrm{MoS}_{2}$ is a $2 \mathrm{D}$ semiconductor, interesting to make ionic liquid-gated devices, such as transistors in which an electrolyte is used to polarize the material and to modulate the density of charge carriers. ${ }^{112,113}$ A parallel- 
plate capacitor was setup, as shown in Fig. 7, with the ionic liquids as the electrolytes and the image-charge constant-potential method representing the polarization of the $\mathrm{MoS}_{2}$ surfaces, at imposed electrostatic potential.

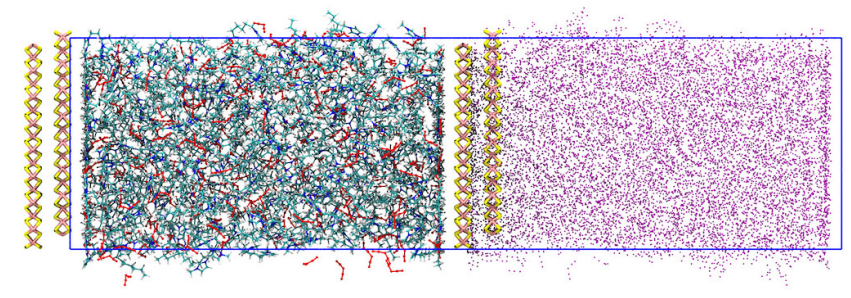

Figure 7: Constant-potential simulation of a substituted imidazolium dicyanamide ionic liquid electrolyte near charged $\mathrm{MoS}_{2}$ surfaces, with the polarization of the material represented by the image charge method.

The effect of the side-chain length on the cations and of different substituents, namely aromatic and hydroxide groups, on the structure of the electrical double layer was analysed, providing details and insights about the orientation of ions and also on the capacitance and on the dynamics of charging the interface.

\section{Conclusions}

Polarizable force fields offer a productive and insightful framework to study systems with ionic liquid phases, be it as solvents, electrolytes, lubricants, confined liquids or coatings, among other possible roles. At present all-atom molecular dynamics simulation strikes an interesting compromise between level of detail and the system size and time scales attainable with reasonable computational cost. This is the mid-level, between quantum chemistry at the bottom, useful to investigate chemical or quasi-chemical phenomena (reactivity, charge transfer) at a local molecular scale, and coarse-grained models at the top, which are suitable for slow, long-range ordered systems such as ionic liquid crystals or polyelectrolytes. We learned over almost two decades that ionic liquids are sophisticated, their balance between Coulomb and van der Waals forces resulting in non-trivial structural and dynamical features. Eutectic solvents, mixtures of salts with molecular compounds, are also complex with strong 
hydrogen bonds playing a major role (as they do in protic ionic liquids). The availability of the general force fields such as the CL\&Pol model, built to be extendable to new compounds and systems without the need for case-by-case parameterization, is expected to contribute to advances in understanding the the physical chemistry of ionic fluids and their interfaces, thus enabling their rational design as better solvents or electrolytes.

\section{Acknowledgement}

The work presented here was supported by the LIQUI2D research project from the French ANR agency (ANR-18-CE09-0018) and by an IDEX Lyon Fellowship (ANR-16-IDEX-005).

\section{References}

(1) Welton, T. Room-Temperature Ionic Liquids. Solvents for Synthesis and Catalysis. Chem. Rev. 1999, 99, 2071-2083.

(2) Zhang, Q.; De Oliveira Vigier, K.; Royer, S.; Jérôme, F. Deep eutectic solvents: syntheses, properties and applications. Chem. Soc. Rev. 2012, 41, 7108-7146.

(3) Smith, E. L.; Abbott, A. P.; Ryder, K. S. Deep Eutectic Solvents (DESs) and Their Applications. Chem. Rev. 2014, 114, 11060-11082.

(4) Hanke, C. G.; Price, S. L.; Lynden-Bell, R. M. Intermolecular potentials for simulations of liquid imidazolium salts. Mol. Phys. 2001, 99, 801-809.

(5) Morrow, T. I.; Maginn, E. J. Molecular Dynamics Study of the Ionic Liquid 1-n-Butyl3-methylimidazolium Hexafluorophosphate. J. Phys. Chem. B 2002, 106, 1280712813.

(6) Liu, Z.; Huang, S.; Wang, W. A Refined Force Field for Molecular Simulation of Imidazolium-Based Ionic Liquids. J. Phys. Chem. B 2004, 108, 12978-12989. 
(7) Canongia Lopes, J. N.; Deschamps, J.; Pádua, A. A. H. Modeling Ionic Liquids Using a Systematic All-Atom Force Field. J. Phys. Chem. B 2004, 108, 2038-2047.

(8) Canongia Lopes, J. N.; Padua, A. A. H. Molecular force field for ionic liquids composed of triflate or bistriflylimide anions. J. Phys. Chem. B 2004, 108, 16893-16898.

(9) Canongia Lopes, J.; Pádua, A. A. H. Molecular Force Field for Ionic Liquids III: Imidazolium, Pyridinium, and Phosphonium Cations; Chloride, Bromide, and Dicyanamide Anions. J. Phys. Chem. B 2006, 110, 19586-19592.

(10) Canongia Lopes, J.; Pádua, A. A. H.; Shimizu, K. Molecular Force Field for Ionic Liquids IV: Trialkylimidazolium and Alkoxycarbonyl-Imidazolium Cations; Alkylsulfonate and Alkylsulfate Anions. J. Phys. Chem. B 2008, 112, 5039-5046.

(11) Sambasivarao, S. V.; Acevedo, O. Development of OPLS-AA Force Field Parameters for 68 Unique Ionic Liquids. J. Chem. Theory Comput. 2009, $\underline{5}, 1038-1050$.

(12) Shimizu, K.; Almantariotis, D.; Gomes, M. F. C.; Pádua, A. A. H.; Canongia Lopes, J. Molecular Force Field for Ionic Liquids V: Hydroxyethylimidazolium, Dimethoxy-2- Methylimidazolium, and Fluoroalkylimidazolium Cations and Bis(Fluorosulfonyl)Amide, Perfluoroalkanesulfonylamide, and Fluoroalkylfluorophosphate Anions. J. Phys. Chem. B 2010, 114, 3592-3600.

(13) Canongia Lopes, J. N.; Pádua, A. A. H. CL\&P: A generic and systematic force field for ionic liquids modeling. Theor. Chem. Acc. 2012, 131, 1129.

(14) Doherty, B.; Zhong, X.; Gathiaka, S.; Li, B.; Acevedo, O. Revisiting OPLS Force Field Parameters for Ionic Liquid Simulations. J. Chem. Theory Comput. 2017, 13, $6131-6145$.

(15) Perkins, S. L.; Painter, P.; Colina, C. M. Molecular Dynamic Simulations and Vibrational Analysis of an Ionic Liquid Analogue. J. Phys. Chem. B 2013, 117, 10250-10260. 
(16) Perkins, S. L.; Painter, P.; Colina, C. M. Experimental and Computational Studies of Choline Chloride-Based Deep Eutectic Solvents. J. Chem. Eng. Data 2014, 59, $3652-3662$.

(17) García, G.; Atilhan, M.; Aparicio, S. The impact of charges in force field parameterization for molecular dynamics simulations of deep eutectic solvents. J. Mol. Liq. 2015, $211,506-514$.

(18) Alcalde, R.; Gutiérrez, A.; Atilhan, M.; Aparicio, S. An experimental and theoretical investigation of the physicochemical properties on choline chloride -Lactic acid based natural deep eutectic solvent (NADES). J. Mol. Liq. 2019, 290, 110916.

(19) Doherty, B.; Acevedo, O. OPLS Force Field for Choline Chloride-Based Deep Eutectic Solvents. J. Phys. Chem. B 2018, 122, 9982-9993.

(20) Ji, Y.; Shi, R.; Wang, Y.; Saielli, G. Effect of the Chain Length on the Structure of Ionic Liquids: From Spatial Heterogeneity to Ionic Liquid Crystals. J. Phys. Chem. B 2013, 117, 1104-1109.

(21) Roy, D.; Maroncelli, M. An Improved Four-Site Ionic Liquid Model. J. Phys. Chem. B 2010, 114, 12629-12631.

(22) Fajardo, O. Y.; Lecce, S. D.; Bresme, F. Molecular Dynamics Simulation of Imidazolium CnMIM-BF4 Ionic Liquids Using a Coarse Grained Force-Field. Phys. Chem. Chem. Phys. 2020, 22, 1682-1692.

(23) Souza, P. C. T.; Alessandri, R.; Barnoud, J.; Thallmair, S.; Faustino, I.; Grünewald, F.; Patmanidis, I.; Abdizadeh, H.; Bruininks, B. M. H.; Wassenaar, T. A.; Kroon, P. C.; Melcr, J.; Nieto, V.; Corradi, V.; Khan, H. M.; Domański, J.; Javanainen, M.; Martinez-Seara, H.; Reuter, N.; Best, R. B.; Vattulainen, I.; Monticelli, L.; Periole, X.; Tieleman, D. P.; de Vries, A. H.; Marrink, S. J. Martini 3: A General Purpose Force Field for Coarse-Grained Molecular Dynamics. Nat Methods 2021, 18, 382-388. 
(24) Vazquez-Salazar, L. I.; Selle, M.; de Vries, A. H.; Marrink, S. J.; Souza, P. C. T. Martini Coarse-Grained Models of Imidazolium-Based Ionic Liquids: From Nanostructural Organization to Liquid-Liquid Extraction. Green Chem. 2020, 22, 7376-7386.

(25) Liu, Z.; Wu, X.; Wang, W. A Novel United-Atom Force Field for Imidazolium-Based Ionic Liquids. Phys. Chem. Chem. Phys. 2006, 8, 1096-1104.

(26) Kirchner, B.; Hollóczki, O.; Canongia Lopes, J. N.; Pádua, A. A. H. Multiresolution Calculation of Ionic Liquids. WIREs Comput. Mol. Sci. 2015, $\underline{5}$, 202-214.

(27) Canongia Lopes, J. N. A.; Pádua, A. A. H. Nanostructural Organization in Ionic Liquids. J. Phys. Chem. B 2006, 110, 3330-3335.

(28) Triolo, A.; Russina, O.; Bleif, H.-J.; Di Cola, E. Nanoscale Segregation in Room Temperature Ionic Liquids. J. Phys. Chem. B 2007, 111, 4641-4644.

(29) Kashyap, H. K.; Annapureddy, H. V. R.; Raineri, F. O.; Margulis, C. J. How Is Charge Transport Different in Ionic Liquids and Electrolyte Solutions? J. Phys. Chem. B 2011, 115, 13212-13221.

(30) Sheridan, Q. R.; Schneider, W. F.; Maginn, E. J. Role of Molecular Modeling in the Development of CO2-Reactive Ionic Liquids. Chem. Rev. 2018, 118, 5242-5260.

(31) Hammond, O. S.; Simon, G.; Gomes, M. C.; Padua, A. A. H. Tuning the Solvation of Indigo in Aqueous Deep Eutectics. J. Chem. Phys. 2021, 154, 224502.

(32) Jorgensen, W. L.; Maxwell, D. S.; Tirado-Rives, J. Development and Testing of the OPLS All-Atom Force Field on Conformational Energetics and Properties of Organic Liquids. J. Am. Chem. Soc. 1996, 118, 11225-11236.

(33) Wang, J.; Wolf, R. M.; Caldwell, J. W.; Kollman, P. A.; Case, D. A. Development and Testing of a General Amber Force Field. J. Comput. Chem. 2004, 25, 1157-1174. 
(34) Vanommeslaeghe, K.; Hatcher, E.; Acharya, C.; Kundu, S.; Zhong, S.; Shim, J.; Darian, E.; Guvench, O.; Lopes, P.; Vorobyov, I.; MacKerell, A. D. CHARMM General Force Field: A Force Field for Drug-like Molecules Compatible with the CHARMM All-Atom Additive Biological Force Fields. J. Comput. Chem. 2010, 31, 671-690.

(35) Tsuzuki, S.; Shinoda, W.; Saito, H.; Mikami, M.; Tokuda, H.; Watanabe, M. Molecular Dynamics Simulations of Ionic Liquids: Cation and Anion Dependence of SelfDiffusion Coefficients of Ions. J. Phys. Chem. B 2009, 113, 10641-10649.

(36) Chaban, V. Polarizability versus mobility: atomistic force field for ionic liquids. Phys. Chem. Chem. Phys 2011, 13, 16055-16062.

(37) Bhargava, B. L.; Balasubramanian, S. Refined potential model for atomistic simulations of ionic liquid [bmim] $\left[\mathrm{PF}_{6}\right]$. J. Chem. Phys. 2007, 127, 114510.

(38) Maginn, E. J. Molecular Simulation of Ionic Liquids: Current Status and Future Opportunities. J. Phys.-Condens. Matter 2009, 21, 373101-373101.

(39) Cui, K.; Yethiraj, A.; Schmidt, J. R. Influence of Charge Scaling on the Solvation Properties of Ionic Liquid Solutions. J. Phys. Chem. B 2019, 123, 9222-9229.

(40) Schröder, C. Comparing reduced partial charge models with polarizable simulations of ionic liquids. Phys. Chem. Chem. Phys. 2012, 14, 3089-3102.

(41) Lemkul, J. A.; Huang, J.; Roux, B.; MacKerell, A. D. An Empirical Polarizable Force Field Based on the Classical Drude Oscillator Model: Development History and Recent Applications. Chem. Rev. 2016, 116, 4983-5013.

(42) Bedrov, D.; Piquemal, J.-P.; Borodin, O.; MacKerell, A. D.; Roux, B.; Schröder, C. Molecular Dynamics Simulations of Ionic Liquids and Electrolytes Using Polarizable Force Fields. Chem. Rev. 2019, 119, 7940-7995. 
(43) Wilson, M.; Madden, P. A. Polarization effects in ionic systems from first principles. J. Phys. Condens. Matter 1993, 5, 2687-2706.

(44) Ren, P.; Ponder, J. W. Consistent treatment of inter- and intramolecular polarization in molecular mechanics calculations. J. Comp. Chem. 2002, 23, 1497-1506.

(45) Ren, P.; Ponder, J. W. Polarizable Atomic Multipole Water Model for Molecular Mechanics Simulation. J. Phys. Chem. B 2003, 107, 5933-5947.

(46) Rick, S. W.; Stuart, S. J. Potentials and Algorithms for Incorporating Polarizability in Computer Simulations; John Wiley \& Sons, Ltd, 2002; Chapter 3, pp 89-146.

(47) Drude, P. The theory of optics; Courier Dover Publications, 1901.

(48) van Maaren, P. J.; van der Spoel, D. Molecular Dynamics Simulations of Water with Novel Shell-Model Potentials. J. Phys. Chem. B 2001, 105, 2618-2626.

(49) Lamoureux, G.; Roux, B. Modeling induced polarization with classical Drude oscillators: Theory and molecular dynamics simulation algorithm. J. Chem. Phys. 2003, $119,3025-3039$.

(50) Lamoureux, G.; MacKerell, A. D.; Roux, B. A simple polarizable model of water based on classical Drude oscillators. J. Chem. Phys. 2003, 119, 5185-5197.

(51) Schmollngruber, M.; Lesch, V.; Schröder, C.; Heuer, A.; Steinhauser, O. Comparing induced point-dipoles and Drude oscillators. Phys. Chem. Chem. Phys. 2015, 17, $14297-14306$.

(52) Rappe, A. K.; Goddard III, W. A. Charge Equilibration for Molecular Dynamics Simulations. J. Phys. Chem. 1991, 95, 3358-3363.

(53) Rick, S. W.; Stuart, S. J.; Berne, B. J. Dynamical fluctuating charge force fields: Application to liquid water. J. Chem. Phys. 1994, 101, 6141-6156. 
(54) Wu, Y.; Li, Y.; Hu, N.; Hong, M. The electronegativity equalization method fused with molecular mechanics: a fluctuating charge and flexible body potential function for [Emim][Gly] ionic liquids. Phys. Chem. Chem. Phys. 2014, 16, 2674-2685.

(55) Bauer, B. A.; Patel, S. Recent Applications and Developments of Charge Equilibration Force Fields for Modeling Dynamical Charges in Classical Molecular Dynamics Simulations. Theor. Chem. Acc. 2012, 131, 1115-1153.

(56) Cavalcante, A. d. O.; Ribeiro, M. C. C.; Skaf, M. S. Polarizability effects on the structure and dynamics of ionic liquids. J. Chem. Phys. 2014, 140, 144108.

(57) Nistor, R. A.; Polihronov, J. G.; Müser, M. H.; Mosey, N. J. A Generalization of the Charge Equilibration Method for Nonmetallic Materials. J. Chem. Phys. 2006, 125, 94108-94111.

(58) Verstraelen, T.; Van Speybroeck, V.; Waroquier, M. The Electronegativity Equalization Method and the Split Charge Equilibration Applied to Organic Systems: Parametrization, Validation, and Comparison. J. Chem. Phys. 2009, 131, 4412044127.

(59) Jiang, W.; Hardy, D. J.; Phillips, J. C.; MacKerell, A. D.; Schulten, K.; Roux, B. High-performance scalable molecular dynamics simulations of a polarizable force field based on classical Drude oscillators in NAMD. J. Phys. Chem. Lett. 2011, 2, 87-92.

(60) Lemkul, J. A.; Roux, B.; van der Spoel, D.; MacKerell Jr., A. D. Implementation of extended Lagrangian dynamics in GROMACS for polarizable simulations using the classical Drude oscillator model. J. Comp. Chem. 2015, 36, 1473-1479.

(61) Dequidt, A.; Devémy, J.; Pádua, A. A. H. Thermalized Drude Oscillators with the LAMMPS Molecular Dynamics Simulator. J. Chem. Info. Model. 2016, 56, 260-268. 
(62) Huang, J.; Lemkul, J. A.; Eastman, P. K.; MacKerell Jr., A. D. Molecular dynamics simulations using the drude polarizable force field on GPUs with OpenMM: Implementation, validation, and benchmarks. J. Comput. Chem. 2018, 39, 1682-1689.

(63) Yan, T.; Burnham, C. J.; Del Pópolo, M. G.; Voth, G. A. Molecular Dynamics Simulation of Ionic Liquids: The Effect of Electronic Polarizability. J. Phys. Chem. B 2004, 108, 11877-11881.

(64) Schröder, C.; Steinhauser, O. Simulating polarizable molecular ionic liquids with Drude oscillators. J. Chem. Phys. 2010, 133, 154511.

(65) Nakano, H.; Yamamoto, T.; Kato, S. A wave-function based approach for polarizable charge model: Systematic comparison of polarization effects on protic, aprotic, and ionic liquids. J. Chem. Phys. 2010, 132, 044106.

(66) Salanne, M.; Siqueira, L. J. A.; Seitsonen, A. P.; Madden, P. A.; Kirchner, B. From molten salts to room temperature ionic liquids: Simulation studies on chloroaluminate systems. Faraday Discuss. 2012, 154, 171-188.

(67) Borodin, O. Polarizable force field development and molecular dynamics simulations of ionic liquids. J. Phys. Chem. B 2009, 113, 11463-11478.

(68) Starovoytov, O. N.; Torabifard, H.; Cisneros, G. A. Development of AMOEBA Force Field for 1,3-Dimethylimidazolium Based Ionic Liquids. J. Phys. Chem. B 2014, 118, $7156-7166$.

(69) Vázquez-Montelongo, E. A.; Vázquez-Cervantes, J. E.; Cisneros, G. A. Current Status of AMOEBA-IL: A Multipolar/Polarizable Force Field for Ionic Liquids. Int. J. Mol. Sci. 2020, 21 .

(70) McDaniel, J. G.; Choi, E.; Son, C. Y.; Schmidt, J. R.; Yethiraj, A. Ab Initio Force Fields for Imidazolium-Based Ionic Liquids. J. Phys. Chem. B 2016, 120, 7024-7036. 
(71) Goloviznina, K.; Canongia Lopes, J. N.; Costa Gomes, M.; Pádua, A. A. H. Transferable, Polarizable Force Field for Ionic Liquids. J. Chem. Theory Comput. 2019, 15, $5858-5871$.

(72) McDaniel, J. G.; Son, C. Y.; Yethiraj, A. Ab Initio Force Fields for Organic Anions: Properties of [BMIM][TFSI], [BMIM][FSI], and [BMIM][OTf] Ionic Liquids. J. Phys. Chem. B 2018, 122, 4101-4114.

(73) Pádua, A. A. H. Resolving Dispersion and Induction Components for Polarisable Molecular Simulations of Ionic Liquids. J. Chem. Phys. 2017, 146, 204501.

(74) Goloviznina, K.; Gong, Z.; Costa Gomes, M. F.; Pádua, A. A. H. Extension of the CL\&Pol Polarizable Force Field to Electrolytes, Protic Ionic Liquids, and Deep Eutectic Solvents. J. Chem. Theory Comput. 2021,

(75) Son, C. Y.; McDaniel, J. G.; Cui, Q.; Yethiraj, A. Conformational and Dynamic Properties of Poly(ethylene oxide) in BMIM+BF4-: A Microsecond Computer Simulation Study Using ab Initio Force Fields. Macromolecules 2018, 51, 5336-5345.

(76) Jeong, K.-j.; McDaniel, J. G.; Yethiraj, A. A Transferable Polarizable Force Field for Urea Crystals and Aqueous Solutions. J. Phys. Chem. B 2020, 124, 7475-7483.

(77) McDaniel, J. G.; Schmidt, J. R. Physically-Motivated Force Fields from SymmetryAdapted Perturbation Theory. J. Phys. Chem. A 2013, 117, 2053-2066.

(78) Watkins, E. K.; Jorgensen, W. L. Perfluoroalkanes: Conformational Analysis and Liquid-State Properties from Ab Initio and Monte Carlo Calculations. J. Phys. Chem. A 2001-04, 105, 4118-4125.

(79) Cornell, W. D.; Cieplak, P.; Bayly, C. I.; Gould, I. R.; Merz, K. M.; Ferguson, D. M.; Spellmeyer, D. C.; Fox, T.; Caldwell, J. W.; Kollman, P. A. A Second Generation 
Force Field for the Simulation of Proteins, Nucleic Acids, and Organic Molecules. J. Am. Chem. Soc. 1995-01, 117, 5179-5197.

(80) Heid, E.; Szabadi, A.; Schröder, C. Quantum mechanical determination of atomic polarizabilities of ionic liquids. Phys. Chem. Chem. Phys. 2018, 20, 10992-10996.

(81) Heid, E.; Heindl, M.; Dienstl, P.; Schröder, C. Additive polarizabilities of halides in ionic liquids and organic solvents. J. Chem. Phys. 2018, 149, 044302.

(82) Son, C. Y.; McDaniel, J. G.; Cui, Q.; Yethiraj, A. Proper Thermal Equilibration of Simulations with Drude Polarizable Models: Temperature-Grouped DualNosé-Hoover Thermostat. J. Phys. Chem. Lett. 2019, 10, 7523-7530.

(83) van Duijnen, P. T.; Swart, M. Molecular and Atomic Polarizabilities: Thole's Model Revisited. J. Phys. Chem. A 1998, 102, 2399-2407.

(84) Noskov, S. Y.; Lamoureux, G.; Roux, B. Molecular dynamics study of hydration in ethanol-water mixtures using a polarizable force field. J. Phys. Chem. B 2005, 109, 6705-6713.

(85) Wang, J.; Cieplak, P.; Cai, Q.; Hsieh, M.-J.; Wang, J.; Duan, Y.; Luo, R. Development of Polarizable Models for Molecular Mechanical Calculations. 3. Polarizable Water Models Conforming to Thole Polarization Screening Schemes. J. Phys. Chem. B 2012, 116, 7999-8008.

(86) Taylor, T.; Schmollngruber, M.; Schröder, C.; Steinhauser, O. The effect of Thole functions on the simulation of ionic liquids with point induced dipoles at various densities. J. Chem. Phys. 2013, 138, 204119.

(87) Thole, B. T. Molecular polarizabilities calculated with a modified dipole interaction. Chem. Phys. 1981, 59, 341-350. 
(88) Jemmer, P.; Wilson, M.; Madden, P. A.; Fowler, P. W. Dipole and quadrupole polarization in ionic systems: Ab initio studies. J. Chem. Phys. 1999, 111, 2038-2049.

(89) Salanne, M.; Madden, P. A. Polarization effects in ionic solids and melts. Mol. Phys. 2011, 109, 2299-2315.

(90) Salanne, M.; Rotenberg, B.; Jahn, S.; Vuilleumier, R.; Simon, C.; Madden, P. A. Including many-body effects in models for ionic liquids. Theor. Chem. Acc. 2012, $\underline{131}, 1143$.

(91) Tang, K. T.; Peter Toennies, J. An improved simple model for the van der Waals potential based on universal damping functions for the dispersion coefficients. J. Chem. Phys. 1984, 80, 3726-3741.

(92) Jeziorski, B.; Moszynski, R.; Szalewicz, K. Perturbation theory approach to intermolecular potential energy surfaces of van der Waals complexes. Chem. Rev. 1994, $\underline{94}, 1887-1930$.

(93) Hohenstein, E. G.; Sherrill, C. D. Wavefunction methods for noncovalent interactions. Wiley Interdiscip. Rev. Comput. Mol. Sci 2011, 2, 304-326.

(94) Lamoureux, G.; Harder, E.; Vorobyov, I. V.; Roux, B.; MacKerell, A. D. A polarizable model of water for molecular dynamics simulations of biomolecules. Chem. Phys. Lett. 2006, 418, 245-249.

(95) Becker, T. M.; Lin, L.-C.; Dubbeldam, D.; Vlugt, T. J. H. Polarizable Force Field for CO2 in M-MOF-74 Derived from Quantum Mechanics. J. Phys. Chem. B 2018, $\underline{122}$, $24488-24498$.

(96) Chandrasekhar, J.; Spellmeyer, D. C.; Jorgensen, W. L. Energy component analysis for dilute aqueous solutions of $\operatorname{lithium}(1+), \operatorname{sodium}(1+)$, fluoride(1-), and chloride(1-) ions. J. Am. Chem. Soc. 1984, 106, 903-910. 
(97) Bodo, E.; Sferrazza, A.; Caminiti, R.; Mangialardo, S.; Postorino, P. A prototypical ionic liquid explored by ab initio molecular dynamics and Raman spectroscopy. J. Chem. Phys. 2013, 139, 144309.

(98) Hayes, R.; Imberti, S.; Warr, G. G.; Atkin, R. The Nature of Hydrogen Bonding in Protic Ionic Liquids. Angew. Chem. Int. Ed. 2013, 52, 4623-4627.

(99) Song, X.; Hamano, H.; Minofar, B.; Kanzaki, R.; Fujii, K.; Kameda, Y.; Kohara, S.; Watanabe, M.; Ishiguro, S. I.; Umebayashi, Y. Structural Heterogeneity and Unique Distorted Hydrogen Bonding in Primary Ammonium Nitrate Ionic Liquids Studied by High-Energy X-Ray Diffraction Experiments and MD Simulations. J. Phys. Chem. B 2012, $\underline{116}, 2801-2813$.

(100) Russina, O.; Mariani, A.; Caminiti, R.; Triolo, A. Structure of a Binary Mixture of Ethylammonium Nitrate and Methanol. J. Solution Chem. 2015-04, 44, 669-685.

(101) Zahn, S.; Thar, J.; Kirchner, B. Structure and Dynamics of the Protic Ionic Liquid Monomethylammonium Nitrate ([CH3NH3][NO3]) from Ab Initio Molecular Dynamics Simulations. J. Chem. Phys. 2010-03-28, 132, 124506.

(102) Zentel, T.; Kühn, O. Properties of Hydrogen Bonds in the Protic Ionic Liquid Ethylammonium Nitrate: DFT versus DFTB Molecular Dynamics. Theor. Chem. Acc. 2017, $\underline{136}, 1-10$.

(103) Gontrani, L.; Bodo, E.; Triolo, A.; Leonelli, F.; D’Angelo, P.; Migliorati, V.; Caminiti, R. The Interpretation of Diffraction Patterns of Two Prototypical Protic Ionic Liquids: A Challenging Task for Classical Molecular Dynamics Simulations. J. Phys. Chem. B 2012, 116, 13024-13032.

(104) Endres, F. Ionic Liquids: Solvents for the Electrodeposition of Metals and Semiconductors. ChemPhysChem 2002, $\underline{3}, 145-154$. 
(105) Macfarlane, D. R.; Forsyth, M.; Howlett, P. C.; Pringle, J. M.; Sun, J.; Annat, G.; Neil, W.; Izgorodina, E. I. Ionic Liquids in Electrochemical Devices and Processes: Managing Interfacial Electrochemistry. Acc. Chem. Res. 2007, 40, 1165-1173.

(106) Armand, M.; Endres, F.; Macfarlane, D. R.; Ohno, H.; Scrosati, B. Ionic-Liquid Materials for the Electrochemical Challenges of the Future. Nat. Mater. 2009-01, $\underline{8}$, $621-629$.

(107) Merlet, C.; Rotenberg, B.; Madden, P. A.; Taberna, P.-L.; Simon, P.; Gogotsi, Y.; Salanne, M. On the Molecular Origin of Supercapacitance in Nanoporous Carbon Electrodes. Nat. Mater. 2012, 11, 306-310.

(108) Reed, S. K.; Lanning, O. J.; Madden, P. A. Electrochemical Interface between an Ionic Liquid and a Model Metallic Electrode. J. Chem. Phys. 2007, 126, 84704-84704.

(109) Wang, Z.; Yang, Y.; Olmsted, D. L.; Asta, M.; Laird, B. B. Evaluation of the Constant Potential Method in Simulating Electric Double-Layer Capacitors. J. Chem. Phys. 2014-11, 141, 184102-184107.

(110) Dwelle, K. A.; Willard, A. P. Constant Potential, Electrochemically Active Boundary Conditions for Electrochemical Simulation. J. Phys. Chem. C 2019, 123, 24095-24103.

(111) Gebbie, M. A.; Smith, A. M.; Dobbs, H. A.; Lee, A. A.; Warr, G. G.; Banquy, X.; Valtiner, M.; Rutland, M. W.; Israelachvili, J. N.; Perkin, S.; Atkin, R. Long Range Electrostatic Forces in Ionic Liquids. Chem. Commun. 2017, 53, 1214-1224.

(112) Perera, M. M.; Lin, M.-W.; Chuang, H.-J.; Chamlagain, B. P.; Wang, C.; Tan, X.; Cheng, M. M.-C.; Tománek, D.; Zhou, Z. Improved Carrier Mobility in Few-Layer MoS2 Field-Effect Transistors with Ionic-Liquid Gating. ACS Nano 2013-05, 7, 44494458. 
(113) Zhao, W.; Bi, S.; Balke, N.; Rack, P. D.; Ward, T. Z.; Kalinin, S. V.; Dai, S.; Feng, G. Understanding Electric Double-Layer Gating Based on Ionic Liquids: From Nanoscale to Macroscale. ACS Appl. Mater. Interfaces 2018, 10, 43211-43218. 